Original Research Article

\title{
Comparative study using routine stains and Immunohistochemistry staining techniques for detection of Helicobacter pylori
}

\author{
Priyadarshini M. M. ${ }^{1}$, Manjunatha Y.A. ${ }^{2}$, Choudhary S. ${ }^{3}$, Suba G $^{4}$ \\ ${ }^{1}$ Dr. Priyadarshini MM, Assistant Professor, Kodagu Institute of Medical Sciences, Madikeri, ${ }^{2}$ Dr. Manjunatha Y.A \\ Professor and HOD, Department of Pathology, Ambedkar Medical College Bangalore, ${ }^{3}$ Dr. Shaista Choudhary, \\ Associate Professor, Department of Pathology, Ambedkar Medical College, Bangalore, ${ }^{4}$ Dr. Suba G, Assistant Professor, \\ Department of Pathology Ambedkar Medical College Bangalore, Karnataka, India.
}

Corresponding Author: D. Priyadarshini MM, Assistant Professor, Kodagu Institute of Medical Sciences, Madikeri. Email: piyadarsh@gmail.com

\begin{abstract}
Introduction: The aim of this study was to evaluate the role of endoscopic biopsies for detection of Helicobacter Pylori using routine staining techniques which included hematoxyline and eosin, Giemsa and Immunohistochemistry. Methods: A prospective study of 3 years in which 53 gastric endoscopic mucosal biopsies were included. These patients had a clinical history of dyspeptic symptoms. 3 staining techniques were used to identify H. Pylori. Hematoxylin and eosin staining was done routinely along with Giemsa and $\mathrm{H}$. Pylori detection using antibodies directed against specific antigens in IHC. The staining pattern of H. Pylori was also studied. Results: In 53 cases studied, modified Giemsa staining is the cheapest and easiest to perform but antibodies directed against specific antigens in IHC proved to be more specific in identifying $\mathrm{H}$. Pylori than other staining techniques. While Giemsa staining was non-specific for other species of Helicobacter IHC was specific for H. Pylori. Spiral type of staining was the most frequent of the staining pattern. Conclusion: Our study highlighted the association of Helicobacter Pylori in patients with functional dyspepsia and proved Immunohistochemistry as gold standard in identifying Helicobacter Pylori with Geimsa being practically applicable in Indian set up keeping the cost factor in mind.
\end{abstract}

Key words: Endoscopy, Helicobacter Pylori, Geimsa, IHC

\section{Introduction}

The discovery of Helicobacter pylori (H. Pylori) and the acceptance of its role in gastric pathophysiology represent a fundamental change in our understanding of gastroduodenal disease. Infection with Helicobacter pylori is carcinogenic to humans (Group 1 carcinogen). H. Pylori infection of the gastric mucosa can be found in majority of population and is associated with a range of pathologies, including chronic gastritis, peptic ulcer disease, atrophic gastritis, gastric MALT lymphoma and gastric adenocarcinoma [1].

Dunn BE et al [2] states that the first isolation of Helicobacter pylori in 1982 by Marshall and Warren ushered in a new era in gastric microbiology. Morphology described by Kusters JG H. pylori is a gram-negative bacterium measuring 2 to $4 \mu \mathrm{m}$ in length and 0.5 to $1 \mu \mathrm{m}$ in width. Although usually spiralshaped, the bacterium can appear as a rod, while

Manuscript received: $20^{\text {th }}$ December 2017

Reviewed: $30^{\text {th }}$ December 2017

Author Corrected: $7^{\text {th }}$ January 2018

Accepted for Publication: $13^{\text {th }}$ January 2018 coccoid shapes appear after prolonged in vitro culture or antibiotic treatment [3]. These coccoid cannot be cultured in vitro and are thought to represent dead cells, although it has been suggested that coccoid forms may represent a viable, nonculturable state. The organism has 2 to 6 unipolar sheathed flagella which often carry a distinctive bulb at the end. The flagella confer motility and allow rapid movement in viscous solutions such as the mucus layer overlying the gastric epithelial cells. In gastric biopsy specimens, H. pylori organisms are 2.5 to $5.0 \mu \mathrm{m}$ long and 0.5 to $1.0 \mu \mathrm{m}$ wide [4].

The histological identification of H.pylori infection is now a widely used means of diagnosis. To achieve this, several staining methods are in use. These include modified Giemsa, Warthin-Starry, Genta, Alcian yellow- toluidine blue method, Triple stain and immunohistochemical $H$. pylori antibody stains. Detection of $H$. pylori by Acridine Orange is highly sensitive, simple and rapid [5]. Immunohistochemistry is the agreed "gold standard" for histology, with a a 


\section{Original Research Article}

highly sensitive and specific staining method. However, the modified Giemsa stain is the method of choice because it is sensitive, cheap, easy to perform, and reproducible [6]. The current study highlights the importance of $\mathrm{H}$ pylori identification using Giemsa and IHC and stressed the significance of simple costeffective stains like Giemsa in identification of $\mathrm{H}$ pylori.

\section{Materials and Methods}

Place of Study- Study was done in Pathology department of Pathology Dr. B R Ambedkar Medical College and Hospital.

Type of Study: Prospective study

Sample Collection- H \& E stained sections of all the cases were reviewed. All endoscopic gastric mucosal biopsies taken from different sites were brought in $10 \%$ buffered formalin and were oriented with submucosa embedded downwards. After overnight fixation in formalin, dehydration done with graded alcohol, clearing in chloroform followed by paraffin embedding and section cutting in rotary microtome. Sections of $3 \mu \mathrm{m}$ thicknesses were made and stained with $\mathrm{H}$ and $\mathrm{E}$, Giemsa and immunohistochemical stains. Sections for IHC were specially taken on poly-L-lysine (PLL) coated slides. Following are the staining technique used for identification of H. Pylori

\section{Sampling Methods}

\section{Giemsa stain: - Procedure:}

1. Bring section down to water

2. Giemsa stain $-5 \mathrm{~min}$
3. Blot

4. Quick dehydration in alcohol

5. Clear in xylene

6. Mount in DPX

Results: H. pylori- Dark Blue, Background -Pink to pale blue

\section{Immunohistochemistry}

1. Formalin fixed paraffin embedded sections are taken

2. Incubate for few hours

3. Sections are deparaffinised 2 changes of xylene and rehydrated with 2 changes of alcohol

4. Primary blocking is done using hydrogen peroxide

5. Heat Antigen retrival by using decloaking chamber

6. Secondary blocking done by bovine serum albumin

7. Incubate with mouse monoclonal primary antibody (specific for H.pylori) for 30 minutes followed by MACH2 secondary antibody

8. 3, 3-diaminobenzidine as chromogen for 5 minutes and Hematoxyline as counter stain

9. Counter stain with hematoxyline

10. Dehydrate in alcohol, clear in xylene and mount it

Inclusion Criteria: Total of 53 patients with symptoms of dyspepsia are selected for the study.

Exclusion Criteria: Resection specimen, tiny tissue with no histological evidence of glands

Stastical Methods- The data was analyzed using SPSS version 20. Microsoft word and Excel have been used to generate graphs, tables.

\section{Results}

The present study was done biopsies on gastric endoscopic mucosal biopsies of patients who presented with symptoms of dyspepsia. 25 of the 53 cases showed positivity for $\mathrm{H}$ pylori which was confirmed with IHC of which 20 (32.7\%) were chronic H. pyloric gastritis. 4 cases which showed carcinoma also were positive for H. pylori.

Of the total 25 cases positive for H. pylori in IHC only 7 cases showed positivity in Hematoxyline and eosin. [Table 1] IHC with specific antibody directed against the antigen showed $100 \%$ specificity. $\mathrm{H}$. pylori in most cases positive in $\mathrm{H} \&$ E were confirmed after IHC was positive in that particular patient, thereby giving $100 \%$ specificity [Figure 1,2].

Table-1: Sensitivity and specificity of H\&E staining technique in comparison with IHC

\begin{tabular}{|c|c|c|c|}
\hline \multirow{2}{*}{ H \& E } & \multicolumn{2}{|c|}{ THC } & \multirow{2}{*}{ Total } \\
\cline { 2 - 4 } & positive & 0 & 7 \\
\hline positive & 7 & 28 & 46 \\
\hline negative & 18 & $\mathbf{2 8}$ & $\mathbf{5 3}$ \\
\hline Total & $\mathbf{2 5}$ & negative \\
\hline
\end{tabular}

Sensitivity $=28 \%$ Specificity $=100 \%$ 


\section{Original Research Article}

Table-2: Sensitivity and specificity of Giemsa staining technique in comparison with IHC.

\begin{tabular}{|c|c|c|c|}
\hline \multirow{2}{*}{ Giemsa } & \multicolumn{2}{|c|}{ IHC } & \multirow{2}{*}{ Total } \\
\cline { 2 - 4 } & Positive & 3 & 23 \\
\hline Positive & 20 & 25 & 30 \\
\hline Negative & 5 & 28 & 53 \\
\hline Total & 25 & Negative \\
\hline
\end{tabular}

Sensitivity $=80 \%$ Specificity $=89.28 \%$

Table-3: H. Pylori Distribution Patterns.

\begin{tabular}{|c|c|c|c|c|}
\hline Equivocal & Luminal & Dot like granular & Spiral & Total \\
\hline $2(8 \%)$ & $4(16 \%)$ & $8(32 \%)$ & $11(44 \%)$ & 25 \\
\hline
\end{tabular}

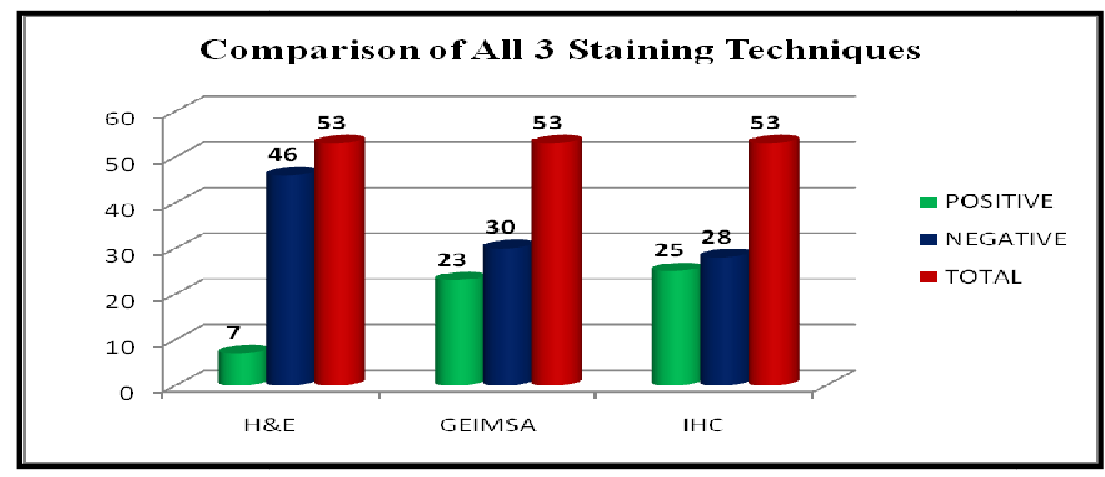

Figure-1: Comparison of All 3 Staining Technique

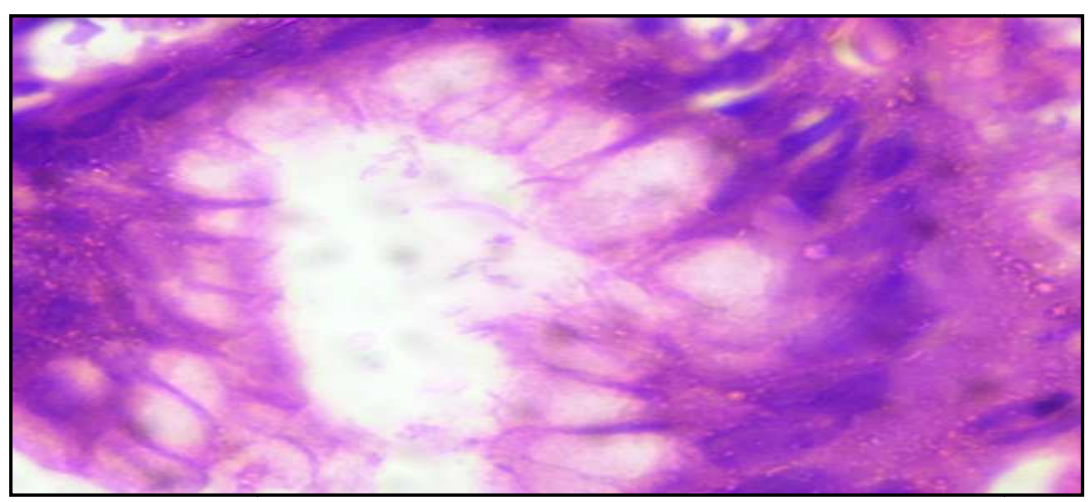

Figure-2: Photomicrograph of H.pylori positive in $\mathrm{H \& E}$ with few small curved organisms (100X)

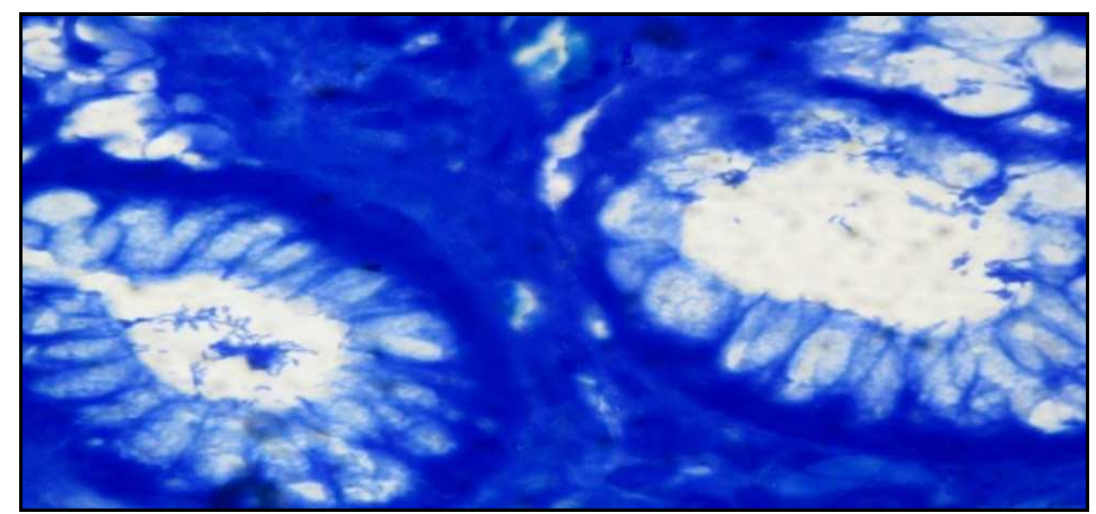

Figure-3: Photomicrograph of H.pylori positive in Giemsa seen as purple or blue curved organisms(100X) 


\section{Original Research Article}

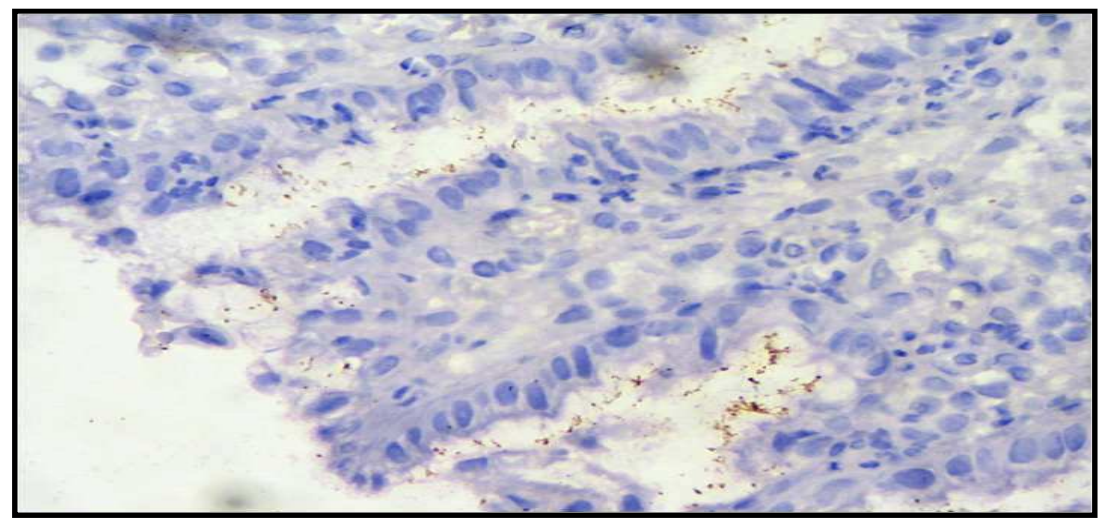

Figure-4: Photomicrograph of H.Pylori Positive in IHC- Spiral Type (chromogen- DAB)
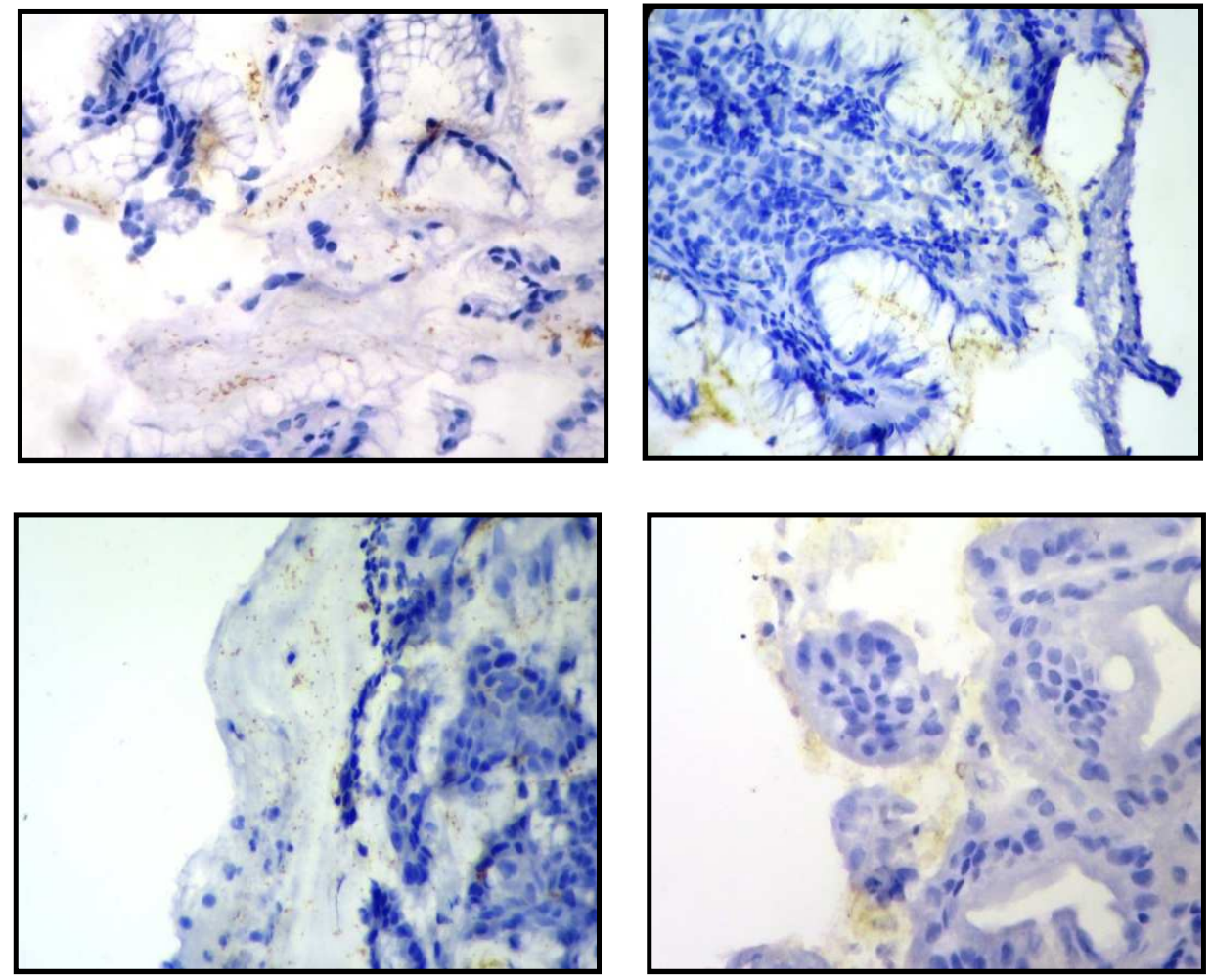

Figure-5: IHC staining patterns: diffuse luminal, spiral, dot like granular and equivocal

As compared to Giemsa which showed positivity in 23 cases of which 3 cases were negative in IHC [Table 2]. This could be due to other Helicobacter pylori like organisms which include Gastrospirillum hominis or other species of Helicobacter (Heilmannii). 5 cases not detected in Giemsa were positive in IHC. This indicates that antibodies directed against specific antigens in IHC are more specific in identifying H. Pylori than other staining techniques [Figure 3,4].

Spiral type of distribution pattern was the most common type seen in 11 cases with small curved bacilli seen. Few cases also showed the cocco bacilli forms of $\mathrm{H}$. pylori [Figure 5] [Table 3]. The bacilli were commonly noted in the luminal surface and more common entrapped within the mucus. They were also noted within the crypts but with much lesser density.

\section{Discussion}

Moayyedi P and Dixon M [7] developed a perspective that in many health care systems, costs are forcing gastroenterologists and other clinicians to review critically their use of endoscopy and the ancillary tests of H. pylori status. Histology is a highly sensitive and specific test for H. pylori but is slow and expensive.
In a study done by Doglioni $\mathrm{C}$ et al [8] $\mathrm{H}$. pylori was detected in 89 biopsies from 48 patients with haematoxylin and eosin; in a further five biopsies (one antral and four fundic) with Giemsa stain, thereby identifying one more H.pylori infected patient. The new silver staining method was positive in all the cases 


\section{Original Research Article}

detected by these two methods and detected three extra infected patients (five more positive biopsies). Immunohistochemistry detected one more positive case (two positive biopsies) not identified by any of the other methods and concluded that the HpSS method proposed is highly sensitive in detecting H.pylori; it is simple and it compares well with other methods used routinely for evaluating gastric biopsies for H.pylori.

By using meta-analysis, Rotimi $\mathrm{O}$ et al [9] observed in most cases, H.pylori can be recognized in a good Haematoxylin and eosin stain. However, the sensitivity of this is low, especially when there are not many bacteria. Although they have shown that the most reliable method is the $H$. pylori immunostain, this is offset by the increased expense of reagents and the time taken for each slide. The extra reliability is unlikely to translate into a cost effective clinical benefit. Giemsa stain with a sensitivity of $98 \%$ was significantly more sensitive than the rest. They have confirmed that the modified Giemsa stain is a reliable, cheap, easy to perform, and convenient histological means of identifying $\mathrm{H}$ pylori in gastric biopsies. Dixon MF et al [10] stated that special stain for H.pylori should be carried out before declaring an inflamed biopsy specimen negative.

Wabinga HR [11] highlighted that 2 cases that showed immunostain could not demonstrate the bacteria but they were identified with modified Giemsa stain while in 5 cases the bacteria were identified by immunostain but not with modified Giemsa stain. The sensitivity of modified Giemsa stain was $85 \%$ (CI 66.5-98.8) while the specificity was $89 \%$ (CI $60.4-97.8$ ). The positive predictive value of modified Giemsa stain was $93 \% \mathrm{CI}$ $75-98.8 \%$ ) while the negative predictive value was $74 \%$ (CI 48.6 - 89.9). The kappa statistic comparing the 2 stains was 0.69 ( $\mathrm{p}$ value 0.00001 ) giving a good agreement between the two tests.

Ashton-Key $\mathrm{M}$ et al [12] detected H.pylori in 14 (37\%) sections stained with haematoxylin and eosin, 21 (55\%) with Giemsa, 23 (61\%) with Warthin-Starry, and 25 $(66 \%)$ stained with the antibody. Seventeen $(45 \%)$ cases were positive on PCR. Immunohistochemistry was positive in all cases in which H.pylori was detected by other methods. Immunohistochemistry using an immunoperoxidase technique following heat induced antigen retrieval for detecting $\mathrm{H}$. pylori in gastric biopsy and resection specimens is highly sensitive and easy to use. The current study showed a total 25 cases positive for H.pylori in IHC only 7 cases showed positivity in Hematoxyline and eosin. As compared to Giemsa which showed positivity in 23 cases of which 3 cases were negative in IHC.
The diagnosis of $\mathrm{H}$. pylori could be performed in hematoxylin and eosin (H\&E) staining, however the specificity can be improved by special stains such as modified Giemsa, Warthin-Starry silver, Genta, and immunohistochemical (IHC) stains. Thus, at least two kinds of stain methods are recommended for diagnosis in practice; H\&E staining is routine and Giemsa stain seems to have advantage over other stains because of its simplicity and consistency. IHC stain may be useful in special situations. However, histology has several limitations, including higher cost, longer turnaround time, dependence on the skills of the operator, and inter observer variability in assessment [13].

The results show that Giemsa stain is superior to HE for histological identification of $\mathrm{Hp}$ in $\mathrm{CG}$. Although $\mathrm{Hp}$ could be identified by $\mathrm{HE}$ stain in the majority of CG cases, a significant number of infected patients may be neglected, regardless the intensity of the inflammatory response. The application of immunohistochemistry for $\mathrm{H}$ pylori identification was first proposed in 1988. Endoscopic biopsies from antral mucosa of dyspeptic patients were used to evaluate $\mathrm{Hp}$ culture as gold standard, and by the peroxidase-antiperoxidase (PAP) method applied in histological sections of formaldehyde fixed biopsy specimens. In the following years, several immunohistochemical methods have been applied for Hp identification and in general, all of them proved to be highly specific and with low inter observer variation. However, this methodology have not been recommended for routine because it is expensive, and in most cases of gastritis with patent inflammatory activity, other easier and cheaper methods could have similar levels of accuracy [14].

H.pylori can be recognized in routine hematoxylin eosin stains, and in most instances that is all that is needed. However, if the density of the organism is low, its detection can be greatly facilitated by the performance of special stains, which include Giemsa, Warthin Starry or Steiner silver stains, the Alcian yellow toluidine blue method, Genta stain or immunohistochemistry [15].

The modified triple stain using Carbol fuchsin, Alcin Blue and $\mathrm{H} \& \mathrm{E}$ is a recently described one for H.pylori detection. Aside from being infected with HP, the other predisposing pathologic condition of gastric cancer is goblet cell intestinal metaplasia. Goblet cell IM may be accentuated by the use of alcian blue staining to identify acid mucin (such as sialomucin and sulfomucin) which is secreted by goblet cells. This can be highlighted by the Alcian Blue component in the triple stain [16]. H.pylori was detected by the 3 special stains-Giemsa, Triple stain and Warthin Starry stain in 29 of the 50 $(58 \%)$ gastric malignancy cases, in 36 of the $50(72 \%)$ 


\section{Original Research Article}

cases stained by Acridine Orange, and in 18/50 (36\%) non malignant cases. Giemsa is cheap and easy to perform. The Alcian blue component of the Triple stain can highlight the areas of intestinal metaplasia and mucin. Warthin Starry stain best detects the curved morphology of H.pylori. Detection of H.pylori by Acridine Orange is highly sensitive, simple and rapid [5].

Giemsa is the most widely used special stain and the accepted gold standard for the histopathological detection of H.pylori because it is cheap, easily available, easily performed and may be repeated without excessive cost on subsequent tissue biopsies during follow-up examinations by a gastroenterologist. The only disadvantage is the lack of contrast between the bacilli and the surrounding tissue [4]. Eleven $(21.6 \%)$ of all specimens included in the study were Helicobacter pylori positive by immunohistochemical methods. Of the Helicobacter pylori positive specimens, the staining pattern was diffuse: Equivocal in $90.9 \%$, nonspecific with a finely granular type concentrated on the luminal surface in $90.9 \%$, dot-like granular in $54.5 \%$, and spiral in $9.1 \%$. [17].

In a study by Riba et al results were classified as either positive or negative for $\mathrm{H}$. pylori. The definition of a positive result was the presence of any stained organisms resembling $\mathrm{H}$. pylori bacteria. The definition of negative was the absence of any stained $\mathrm{H}$. pylorilike bacteria. The typical morphology of $\mathrm{H}$. pylori in biopsy specimens is a comma or S-shaped bacillus that is 2.5-4.0 microns long and 0.5-1.0 microns thick. Patients treated with antibiotics prior to gastric biopsy may demonstrate a markedly reduced number of organisms, and atypical coccoid forms of the organism may be present [18].

In their study Rotimi et al stated that in immunostained preparations, the organisms including coccoid forms, become more prominent [9]. They found that by using heating method for antigen retrieval rather than trypsin, the problem of excessive background staining of epithelium and mucus, seen in IHC stain can be overcome. According to them, immunoperoxidase method is easy to use, less demanding than Warthin Starry staining, and that it produces reliable results, which are easy to interpret. Low numbers or even single organisms, often difficult to detect using traditional stains, are easily identified in immunostained sections $[19,9]$.

In a study by Patnayak et al of the 29 cases, $26(32.9 \%)$ showed presence of $\mathrm{H}$. pylori on $\mathrm{H}$ and $\mathrm{E}$, Giemsa and WS stains, whereas $49(62.0 \%)$ cases demonstrated $\mathrm{H}$. pylori on IHC stain. We conclude that $\mathrm{H}$. pylori detection by IHC has advantage over routine $\mathrm{H}$ and $\mathrm{E}$ staining. However, in the developing countries with financial constraints, routine $\mathrm{H}$ and $\mathrm{E}$ staining in combination with special staining are fairly reliable in demonstrating H. pylori.

The current study showed Spiral type of distribution pattern was the most common type seen in 11 cases with small curved bacilli seen. The cocco bacilli forms observed as dot like granularity could be because of antibiotics influence prior to gastric biopsy.

\section{Conclusion}

This study highlighted the association of Helicobacter Pylori in patients with functional dyspepsia and proving Immunohistochemistry being gold standard in identifying Helicobacter Pylori with Geimsa being practically applicable in Indian set up keeping the cost factor in mind. With careful examination even hematoxylin and eosin stain can show H.Pylori positivity but the density of the organism plays an important role in detection with such staining. The giemsa stain shows positivity in specimens nearly close to immunohistochemical identification and thereby proving its effectiveness.

This study has appraised the role and significance of Giemsa stain in evaluation of Helicobacter Pylori.

Funding: Nil, Conflict of interest: None initiated Permission from IRB: Yes

\section{References}

1. Dyspepsia at Taber's Medical Dictionary. $20^{\text {th }}$ ed. Philadelphia:F.A.Davis Company;2005.Dyspepsia; p.651

2. Dunn BE, Cohen H, Blaser MJ. Helicobacter pylori. Clin Microbiol Rev. 1997 Oct;10(4):720-41

3.Kusters JG, Gerrits MM,Van Strijp JA, Vandenbroucke-Grauls CM. Coccoid forms of Helicobacter pylori are the morphologic manifestation of cell death. Infect Immun. 1997 Sep;65(9):3672-9.

4. Anim JT , Al-Sobkie N, Prasad A, et al. Assessment of different methods for staining Helicobacter pylori in endoscopic gastric biopsies. DOI:10.1078/S0065-1281 (04)70022-7

5. Sulakshana MS, Siddiq M, Ahmed Raghupathi AR. A histopathological study of association of Helicobacter pylori with gastric Malignancies, Int. J Curr Res Aca Rev. 2015; 3(3):10-28. 
6. Schistosomes, liver flukes and Helicobacter pylori. IARC Working Group on the Evaluation of Carcinogenic Risks to Humans. Lyon, 7-14 June 1994. IARC Monogr EvalCarcinogRisksHum.1994;61:1-241

7. Moayyedi P, Dixon MF. Any role left for invasive tests? Histology in clinical practice. Gut. 1998 Jul;43 Suppl 1:S51-5.

8. Doglioni C, Turrin M, Macrì E, et al. HpSS: a new silver staining method for Helicobacter pylori. J Clin Pathol. 1997 Jun;50(6):461-4.

9. Rotimi O, Cairns A, Gray S,v et al. Histological identification of Helicobacter pylori: comparison of staining methods. J Clin Pathol. 2000 Oct;53(10):756-9

10. Dixon MF, Genta RM, Yardley JH, Correa P. Classification and grading of gastritis. The updated Sydney System. International Workshop on the Histopathology of Gastritis, Houston 1994. Am J Surg Pathol. 1996 Oct;20(10):1161-81.

11. Wabinga HR. Comparison of immunohistochemical and modified Giemsa stains for demonstration of Helicobacter pylori infection in an African population. Afr Health Sci. 2002 Aug;2(2):52-5.

12. Ashton-Key M, Diss TC, Isaacson PG. Detection of Helicobacter pylori in gastric biopsy and resection specimens. J Clin Pathol. 1996 Feb;49(2):107-11.

13. Lee JY, Kim N. Diagnosis of Helicobacter pylori by invasive test: histology. 10.3978/j.issn.2305-5839.2014. 11.03 .

\section{Original Research Article}

14. Histological identification of H. pylori stained by hematoxylin-eosin and Giemsa: review for quality control J Bras Patol Med Lab, v. 51, n. 2, p. 108-112, April 2015 Marcela S. Boldt ${ }^{1}$; Rivelle D. Pereira ${ }^{1}$; Alfredo J. A. Barbosa ${ }^{2}$

15. Rosai J. Gastrointestinal tract-Stomach. Rosai and Ackerman s Surgical Pathology. Missouri: $9^{\text {th }}$ ed. Elsevier Inc; 2009: 650- 684.

16. Yodavudh S, Tangjitgamol S, Puangsa S. Mixture of carbol fuchsin and alcian blue staining of gastric tissue for the identification of H.pylori and goblet cell intestinal metaplasia. Southeast Asian J Top Med Pub health; Jul 2008, 39 (4): 656-666.

17. Soylu A, Ozkara S, Alıs H, et al. Immunohistochemical testing for Helicobacter Pylori existence in neoplasms of the colon. BMC Gastroenterology. 2008; 8:35. doi:10.1186/1471-230X-8-35. Riba AK, Ingeneri TJ, Strand CL. Improved histologic identification of Helicobacter pylori by immunohisto-chemistry using a new Novocastra monoclonal antibody. Lab Med [Internet]. 2011;42(1):35-9.

18. Jhala NC, Siegal GP, Klemm K, et al. Infiltration of Helicobacter pylori in the gastric mucosa. DOI: 10 . 1309/YDTX-KE06-XHTH-FNP2

19. Patnayak K, Reddy V, Jena A, Rukmangadha N, Panhasarathy S, Reddy MK. Utility of immunohistochemistry in demonstrating Helicobacter pylori. Oncol Gastroenterol Hepatol. 2015;4:4-7.

\section{How to cite this article?}

Priyadarshini M. M, Manjunatha Y.A, Choudhary S, Suba G. Comparative study using routine stains and Immunohistochemistry staining techniques for detection of Helicobacter pylori. Trop J Path Micro 2018;4(3):288294.doi:10. 17511/jopm.2018.i3.09 